Discrete Comput Geom 36:489-498 (2006)

DOI: $10.1007 / \mathrm{s} 00454-006-1254-3$

Geometry

\title{
Some Incidence Theorems and Integrable Discrete Equations*
}

\author{
Vsevolod E. Adler \\ Institut für Mathematik, Technische Universität Berlin, \\ Str. des 17. Juni 136, 10623 Berlin, Germany \\ adler@itp.ac.ru
}

\begin{abstract}
Several incidence theorems of planar projective geometry are considered. It is demonstrated that generalizations of the Pascal theorem due to Möbius give rise to the double cross-ratio equation and the Hietarinta equation. The construction corresponding to the double cross-ratio equation is a reduction to a conic section of some planar configuration $\left(20_{3} 15_{4}\right)$. This configuration provides a correct definition of the multi-dimensional quadrilateral lattices on the plane.
\end{abstract}

\section{Introduction}

Accordingly to [1] and [8], an $m$-dimensional partial difference equation $F[x]=0$, $x: \mathbb{Z}^{m} \rightarrow \mathbb{R}^{d}$, is called integrable if it can be self-consistently imposed on each $m$ dimensional sublattice in $\mathbb{Z}^{m+1}$. Assume that this equation can be interpreted as a geometric construction which defines some elements of a figure by the other ones. This may be a figure not necessarily in $\mathbb{R}^{d}$, for example, $x$ may play the role of the parameter on some manifold. Then the integrability means that some complex figure exists which contains several copies of our basic figure, and this complex figure can be constructed by the given elements in several ways. In other words, the self-consistency property is expressed geometrically as some incidence theorem.

In some examples, the construction of the basic figure is itself possible due to an incidence, so that already the equation $F[x]=0$ is equivalent to some incidence theorem. This low-level incidence occurs when our equation is a reduction in some more general equation, or, geometrically, our construction is a particular case of some more general construction.

\footnotetext{
* This research was supported by the Alexander von Humboldt Foundation, and was done while the author was on leave from L.D. Landau Institute for Theoretical Physics, Chernogolovka, Russia.
} 
The present paper illustrates these notions by the examples of the double cross-ratio equation [9], the Hietarinta equation [4], and the quadrilateral lattices [3]. Recall that the double cross-ratio, or the discrete Schwarz-BKP equation appears in the theory of Bäcklund transformations for the (2+1)-dimensional sine-Gordon and Nizhnik-VeselovNovikov equations and is equivalent to the Hirota-Miwa equation and the star-triangle map. Its applications in geometry were found in [5] and [6]. Quadrilateral lattices can be considered as the discrete analog of the conjugated nets [3] and are the object of intensive study in the modern theory of integrable systems and discrete geometry (see, for example, the recent review by Bobenko et al. [2]).

We start in Section 2 from the Möbius theorem on the polygons inscribed in a conic section and Theorem 2 which is its modification. Section 3 is devoted to the analytical description of the figures under consideration. In Sections 4 and 6 we consider in more detail the particular cases of these theorems, corresponding to the double cross-ratio and Hietarinta equations.

In Section 5 we show that the double cross-ratio equation is a reduction of some more general mapping which corresponds geometrically to some planar configuration with the symbol $\left(20_{3} 15_{4}\right)$. In turn, this configuration is just the projection on the plane of the elementary cell of the quadrilateral lattice. Integrability of quadrilateral lattices in $\mathbb{R}^{3}$, proved in [3], implies the integrability of this mapping on the plane, and then reduction to the conic section proves the integrability of the double cross-ratio equation.

In brief, the content of the paper is illustrated by the following diagram:

$$
\begin{array}{cc}
\text { Möbius theorem } \stackrel{N=3}{\longrightarrow} \text { Pascal theorem } \stackrel{N=3}{\longleftarrow} \text { Theorem } 2 \\
\qquad N=4 & \downarrow N=4 \\
\text { double cross-ratio equation (6) } & \text { Hietarinta equation (10) } \\
\uparrow \text { reduction to the conic section } &
\end{array}
$$

Theorem 4, quadrilateral lattices on the plane

$$
\begin{gathered}
\uparrow \text { projection } \\
\text { quadrilateral lattices in } \mathbb{R}^{d}
\end{gathered}
$$

\section{Generalizations of the Pascal Theorem}

Recall that, accordingly to the Pascal theorem, (the prolongations of) the opposite sides of a hexagon inscribed in a conic section meet on a straight line. In 1847 Möbius [7] found the following generalizations of this theorem (see Fig. 1):

1. let a $(4 n+2)$-gon be inscribed in a conic section and let $2 n$ pairs of its opposite sides meet on a straight line, then the same is true for the remaining pair;

2. let two $2 n$-gons be inscribed in a conic section and let $2 n-1$ pairs of their corresponding sides meet on a straight line, then the same is true for the remaining pair. 

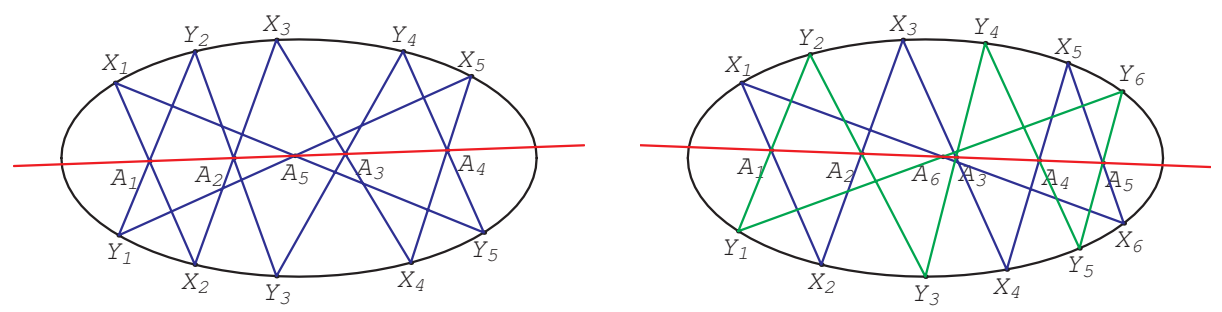

Fig. 1. Möbius theorem, $N=5,6$.

In order to fix the notations, we reformulate this as follows.

Theorem 1 (Möbius). Let $X_{1}, Y_{1}, \ldots, X_{N}, Y_{N}$ be points on a conic section. Consider the intersection points $A_{j}=X_{j} X_{j+1} \cap Y_{j} Y_{j+1}, j=1, \ldots, N-1$, and

$$
A_{N}= \begin{cases}X_{N} Y_{1} \cap Y_{N} X_{1} & \text { if } \quad N=2 n+1 \\ X_{N} X_{1} \cap Y_{N} Y_{1} & \text { if } \quad N=2 n\end{cases}
$$

If all of these points except possibly one are collinear then the same is true for the remaining point.

The proof by Möbius (based on the Gergonne proof of the Pascal theorem) is very simple. Consider the projective transformation of the plane which maps the conic section into a circle and sends the line of intersections to infinity. Then the statement is that if all pairs of the opposite (resp. corresponding) sides except for possibly one are parallel then this is true for the remaining pair as well. This easily follows from the fact that a pair of parallel chords cuts off equal arcs of the circle with opposite orientation and vice versa: $X X^{\prime} \| Y Y^{\prime} \Leftrightarrow \widehat{X Y}=-\widehat{X}^{\prime} Y^{\prime}$. The change of orientation explains the difference between the cases of odd and even $N$. (Possibly, this was the first step on the way to the invention of the Möbius band, in 1861?)

Another proof can be obtained by applying the Pascal theorem to some sequence of hexagons. Assume that we have to prove the collinearity of the last point $A_{N}$. Consider the hexagons $X_{1} X_{2} X_{3} Y_{1} Y_{2} Y_{3}, X_{1} Y_{3} Y_{4} Y_{1} X_{3} X_{4}, X_{1} X_{4} X_{5} Y_{1} Y_{4} Y_{5}$, and so on. On each step we prove that some new intersection point is collinear: first, the point $A^{\prime}=X_{1} Y_{3} \cap Y_{1} X_{3}$, then $A^{\prime \prime}=X_{1} X_{4} \cap Y_{1} Y_{4}, A^{\prime \prime \prime}=X_{1} Y_{5} \cap Y_{1} X_{5}$, and so on until we come to the point $A_{N}$.

The Möbius theorem admits several variations which can be proved by the same reasoning. One of them is given by the following statement (see Fig. 2).

Theorem 2. Let polygon $U X_{1} \cdots X_{N} V Y_{N} \cdots Y_{1}$ be inscribed in a conic section. Consider the intersection points $A_{j}=X_{j} X_{j+1} \cap Y_{j} Y_{j+1}, j=1, \ldots, N-1$, and

$$
\begin{array}{llll}
B=U X_{1} \cap Y_{2 n} V, & C=U Y_{1} \cap X_{2 n} V & \text { if } \quad N=2 n, \\
B=U X_{1} \cap X_{2 n+1} V, & C=U Y_{1} \cap Y_{2 n+1} V & \text { if } \quad N=2 n+1 .
\end{array}
$$

If all of these points except possibly one are collinear then the same is true for the remaining point. 

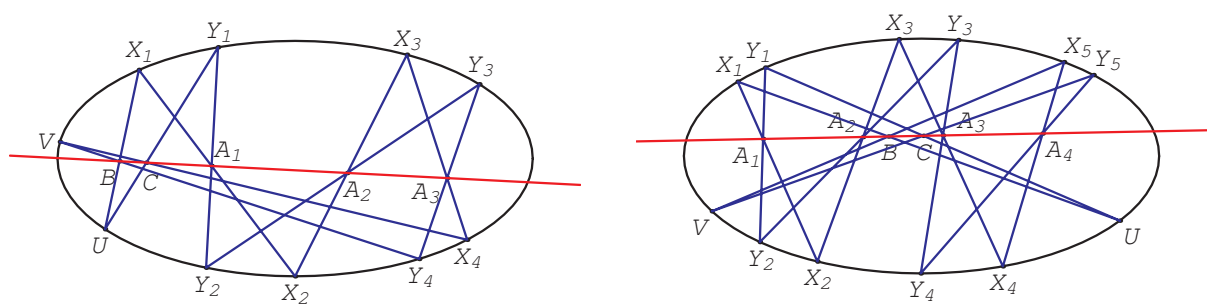

Fig. 2. Theorem 2, $N=4,5$.

\section{The Cross-Ratio Lattice}

Here we consider the system of algebraic equations which is equivalent to the collinearity of the intersection points. In this language, Theorems 1 and 2 mean that any equation of this system is a consequence of all the others. The derivation of the system is based on Lemma 3 below. The notation $(a, b, c, d)=(a-b) /(b-c) \cdot(c-d) /(d-a)$ for the cross-ratio is used and, more general, the multi-ratio is denoted as

$$
(a, b, \ldots, c, d)=(a-b) /(b-\cdots) \cdots(c-d) /(d-a) .
$$

\section{Lemma 3.}

1. Let the broken lines $X_{1} X_{2} X_{3} X_{4}$ and $Y_{1} Y_{2} Y_{3} Y_{4}$ be inscribed into a conic section $\mathcal{C}$, and let $x_{j}, y_{j}$ be the corresponding values of a rational parameter on $\mathcal{C}$. Then the collinearity of the intersection points $A_{j}=X_{j} X_{j+1} \cap Y_{j} Y_{j+1}, j=1,2,3$, is equivalent to the equation

$$
\left(x_{1}, y_{2}, x_{3}, y_{4}\right)=\left(y_{1}, x_{2}, y_{3}, x_{4}\right) .
$$

2. Let the broken lines $Y_{2} Y_{1} U X_{1} X_{2}$ and $P V Q$ be inscribed into a conic section $\mathcal{C}$, and let $x_{j}, y_{j}, u, v, p, q$ be the corresponding values of a rational parameter on $\mathcal{C}$. Then the collinearity of the intersection points

$$
A=X_{1} X_{2} \cap Y_{1} Y_{2}, \quad B=U X_{1} \cap P V, \quad C=U Y_{1} \cap Q V
$$

is equivalent to the equation

$$
\left(p, u, q, x_{2}, y_{1}, v, x_{1}, y_{2}\right)=1 .
$$

Proof. Since all nondegenerate conic sections are equivalent modulo projective transformations of the plane, and all birational parametrizations of the conic section are Möbius-equivalent, it is sufficient to check the formulae for some parametrization of some conic section, for example for the parabola $X=\left(x: x^{2}: 1\right)$. This is a straightforward computation.

It is easy to see that the Möbius theorem corresponds to the system

$$
\left(x_{j}, y_{j+1}, x_{j+2}, y_{j+3}\right)=\left(y_{j}, x_{j+1}, y_{j+2}, x_{j+3}\right), \quad j=1, \ldots, N-2,
$$


with the periodic boundary conditions

$$
\begin{array}{lll}
x_{N+1}=x_{1}, & y_{N+1}=y_{1}, & N=2 n, \\
x_{N+1}=y_{1}, & y_{N+1}=x_{1}, & N=2 n+1 .
\end{array}
$$

In particular, for $N=3$ the system turns into the equation $\left(x_{1}, y_{2}, x_{3}, x_{1}\right)=\left(y_{1}, x_{2}\right.$, $\left.y_{3}, y_{1}\right)$ which is the identity $\infty=\infty$ (Pascal theorem).

For $N=4$ the system consists of (1) and $\left(x_{2}, y_{3}, x_{4}, y_{1}\right)=\left(y_{2}, x_{3}, y_{4}, x_{1}\right)$ which are obviously equivalent.

In the general case we obtain one more proof of the Möbius theorem by checking that the last equation of the system follows from the others. To this end, notice that the arguments of cross-ratios can be interchanged in arbitrary order, simultaneously in leftand right-hand sides, and $(a, b, c, d) /(a, b, c, e)=(a, e, c, d)$.

This allows us to eliminate $x_{2}, y_{2}$ from the first and second equations of the system (2), resulting in $\left(x_{1}, x_{3}, y_{4}, x_{5}\right)=\left(y_{1}, y_{3}, x_{4}, y_{5}\right)$. Geometrically, this means that the point $A^{\prime}=X_{1} Y_{3} \cap Y_{1} X_{3}$ is collinear to $A_{3}, A_{4}$, so that we follow the proof by recursively applying Pascal's theorem as described in the previous section. In the next step, eliminating $x_{3}, y_{3}$ from this new equation and the third equation of the system results in the equation $\left(x_{1}, y_{4}, x_{5}, y_{6}\right)=\left(y_{1}, x_{4}, y_{5}, x_{6}\right)$ which is equivalent to the collinearity of $A^{\prime \prime}, A_{4}, A_{5}$. We repeat this procedure until we come to the equation $\left(x_{1}, x_{N-2}, y_{N-1}, x_{N}\right)=\left(y_{1}, y_{N-2}, x_{N-1}, y_{N}\right)$ if $N$ is odd or $\left(x_{1}, y_{N-2}, x_{N-1}, y_{N}\right)=$ $\left(y_{1}, x_{N-2}, y_{N-1}, x_{N}\right)$ if $N$ is even, as required.

Analogously, Theorem 2 corresponds to the system

$$
\begin{aligned}
& \left(x_{j}, y_{j+1}, x_{j+2}, y_{j+3}\right)=\left(y_{j}, x_{j+1}, y_{j+2}, x_{j+3}\right), \quad j=1, \ldots, N-3, \\
& \left\{\begin{array}{c}
\left(y_{N}, u, x_{N}, x_{2}, y_{1}, v, x_{1}, y_{2}\right)=1 \\
\left(y_{1}, v, x_{1}, x_{N-1}, y_{N}, u, x_{N}, y_{N-1}\right)=1
\end{array} \quad \text { if } \quad N=2 n,\right. \\
& \left\{\begin{array}{c}
\left(x_{N}, u, y_{N}, x_{2}, y_{1}, v, x_{1}, y_{2}\right)=1 \\
\left(y_{1}, v, x_{1}, y_{N-1}, x_{N}, u, y_{N}, x_{N-1}\right)=1
\end{array} \quad \text { if } \quad N=2 n+1,\right.
\end{aligned}
$$

where (4) or (5) are equivalent to the collinearity of the points $B, C, A_{1}$ and $B, C, A_{N}$. The ratio of (4) is equivalent to the equation $\left(x_{1}, y_{2}, x_{N-1}, y_{N}\right)=\left(y_{1}, x_{2}, y_{N-1}, x_{N}\right)$ while the ratio of (5) is equivalent to $\left(x_{1}, y_{2}, y_{N-1}, x_{N}\right)=\left(y_{1}, x_{2}, x_{N-1}, y_{N}\right)$. In both cases, this can be proved to be a consequence of (3).

\section{The Double Cross-Ratio Equation}

Consider in more detail the Möbius theorem at $N=4$. It says that if three pairs of the corresponding sides of inscribed quadrilaterals $X_{1} X_{2} X_{3} X_{4}$ and $Y_{1} Y_{2} Y_{3} Y_{4}$ meet on a straight line, then the same is true for the fourth pair. As we have seen in the previous section, the figure under consideration is governed by (1):

$$
\left(x_{1}, y_{2}, x_{3}, y_{4}\right)=\left(y_{1}, x_{2}, y_{3}, x_{4}\right) .
$$

Due to the transformation properties of cross-ratio this equation can be rewritten in several equivalent forms, so that it also provides the collinearity of several other quadruples of the intersection points (see Fig. 3 where one of these additional lines is shown). 


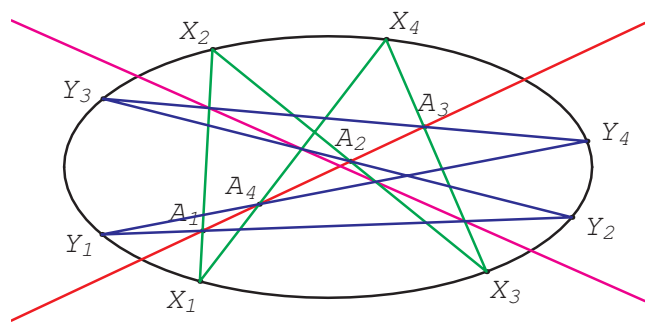

Fig. 3. Intersections in a pair of quadrilaterals.

In particular, (1) is equivalent to $\left(x_{1}, x_{3}, y_{2}, y_{4}\right)=\left(x_{4}, x_{2}, y_{3}, y_{1}\right)$ and in this form it express the collinearity of the intersection points of the corresponding sides of the quadrilaterals $X_{1} X_{2} Y_{2} Y_{1}$ and $X_{4} X_{3} Y_{3} Y_{4}$. Another equivalent form $\left(x_{1}, x_{3}, y_{4}, y_{2}\right)=$ $\left(x_{2}, x_{4}, y_{3}, y_{1}\right)$ corresponds to the quadrilaterals $X_{1} X_{4} Y_{4} Y_{1}$ and $X_{2} X_{3} Y_{3} Y_{2}$.

In order to make the symmetry between these three forms more explicit, we consider the quadrilaterals $X_{1} X_{2} X_{3} X_{4}$ and $Y_{1} Y_{2} Y_{3} Y_{4}$ as the opposite faces of a combinatorial cube and rename them $X X_{1} X_{12} X_{2}$ and $X_{3} X_{13} X_{123} X_{23}$, respectively. In this notation subscripts correspond to the coordinate shifts $T_{i}: X \rightarrow X_{i}$ and their order is unessential, so that $X_{i j}$ and $X_{j i}$ denote the same point. This renumeration brings us to the double cross-ratio equation [5], [9]

$$
\left(x, x_{12}, x_{13}, x_{23}\right)=\left(x_{123}, x_{3}, x_{2}, x_{1}\right) .
$$

This equation is invariant with respect to any interchange of subscripts and therefore it express the collinearity of the intersection points of the corresponding edges for any pair of the opposite faces of the combinatorial cube.

The double cross-ratio equation is known to be integrable in the sense that it can be selfconsistently embedded into a multi-dimensional lattice [1]. This means the following. Consider (6) as a partial difference equation in $\mathbb{Z}^{3}$, so that $x=x\left(n_{1}, n_{2}, n_{3}\right), x_{1}=$ $x\left(n_{1}+1, n_{2}, n_{3}\right)$, and so on. Obviously, a generic solution $x: \mathbb{Z}^{3} \rightarrow \mathbb{C P}^{1}$ is uniquely defined by the initial data on the coordinate planes $n_{i}=0$. Now consider the mapping $x: \mathbb{Z}^{M} \rightarrow \mathbb{C P}^{1}, M>3$, governed by the equation

$$
\left(x, x_{i j}, x_{i k}, x_{j k}\right)=\left(x_{i j k}, x_{k}, x_{j}, x_{i}\right)
$$

for any three-dimensional sublattice. It turns out that such a mapping is also computed from the initial data on the coordinate planes without any contradictions. In order to prove this, it is sufficient to check that if the values $x_{123}, x_{124}, x_{134}, x_{234}$ are found from (7) then the equations

$$
\left(x_{l}, x_{i j l}, x_{i k l}, x_{j k l}\right)=\left(x_{i j k l}, x_{k l}, x_{j l}, x_{i l}\right), \quad\{i, j, k, l\}=\{1,2,3,4\},
$$

define one and the same value $x_{1234}$ as a function on the initial data $x, x_{1}, \ldots, x_{34}$. This property is called four-dimensional consistency. The consistency in the whole $\mathbb{Z}^{M}$ follows. In the next section we will see that four-dimensional consistency of the double cross-ratio equation is inherited from some more general construction. 


\section{Quadrilateral Lattice on the Plane}

The double cross-ratio equation defines the mapping from seven points into one point on the conic section. If $X, X_{1}, \ldots, X_{23}$ are given then $X_{123}$ is constructed by means of two most elementary geometric operations only, drawing a line through two points and finding the intersection of two lines:

$$
\begin{gathered}
A_{1}^{3}=X X_{1} \cap X_{3} X_{13}, \quad A_{2}^{3}=X X_{2} \cap X_{3} X_{23} \\
A_{12}^{3}=X_{2} X_{12} \cap A_{1} A_{2}, \quad A_{21}^{3}=X_{1} X_{12} \cap A_{1} A_{2} \\
X_{123}=A_{12}^{3} X_{23} \cap A_{21}^{3} X_{13} .
\end{gathered}
$$

This can be considered also as the mapping from seven points into one point on the plane. The Möbius theorem guarantees that if initial data lie on a conic section then $X_{123}$ lies on it as well. Moreover, in this case $X_{123}$ does not depend on the interchanging of subscripts in (8), due to the symmetry properties of (6), so that three mappings corresponding to the different pairs of the opposite faces actually coincide. The natural question arise: Is this true only for reductions to conic sections, or also for the generic initial data? The answer is given by the following theorem (see Fig. 4).

Theorem 4. Consider a combinatorial cube on the plane. If, for some pair of opposite faces, the intersection points of the corresponding edges are collinear, then the same is true for any other pair.

Proof. As in the case of the Desargues theorem, the proof is obtained by constructing a three-dimensional figure for which the original one is a planar projection. Denote the

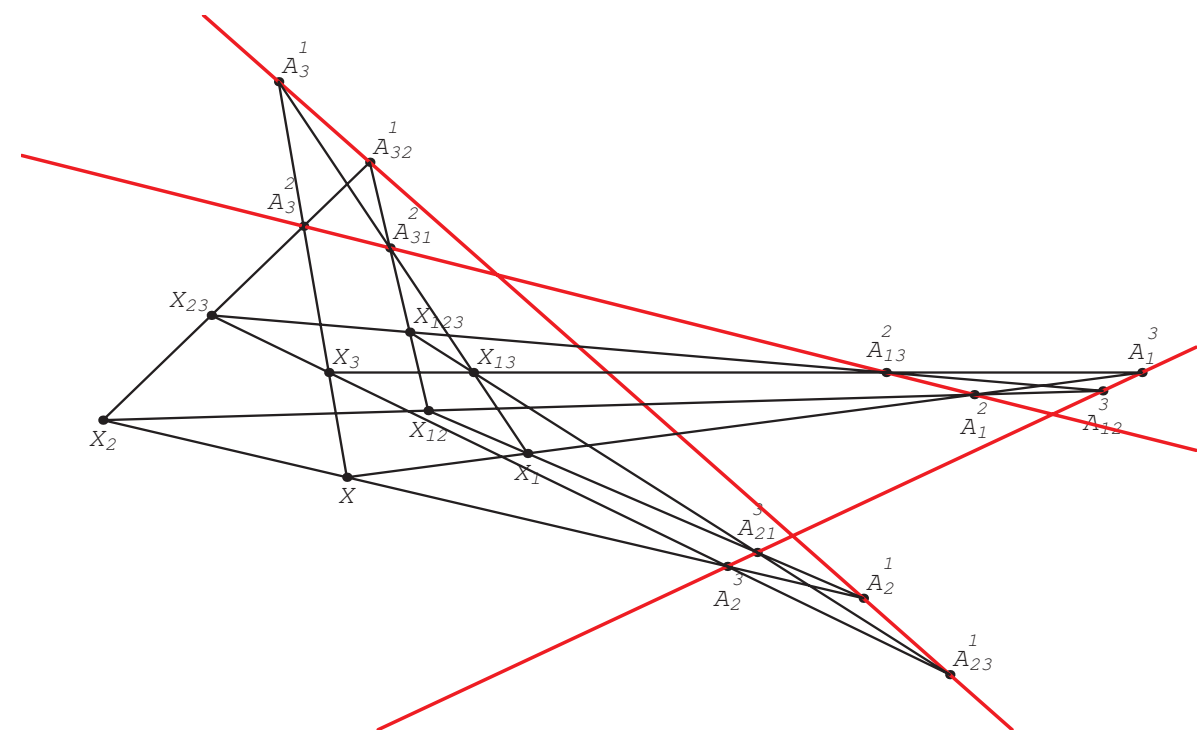

Fig. 4. Theorem 4 . 
intersection points as follows:

$$
A_{i}^{k}=X X_{i} \cap T_{k}\left(X X_{i}\right), \quad A_{i j}^{k}=T_{j}\left(X X_{i}\right) \cap T_{j} T_{k}\left(X X_{i}\right), \quad\{i, j, k\}=\{1,2,3\},
$$

and assume that the points $A_{1}^{3}, A_{2}^{3}, A_{12}^{3}, A_{21}^{3}$ are collinear. Let $\alpha$ be the plane of the figure and let $\alpha^{\prime}$ be some other plane through the line $A_{1}^{3} A_{2}^{3}$. Choose some point $O$ outside both planes and define the face $X_{3}^{\prime} X_{13}^{\prime} X_{123}^{\prime} X_{23}^{\prime}$ as the projection of the face $X_{3} X_{13} X_{123} X_{23}$ from $O$ onto $\alpha^{\prime}$. All faces of the combinatorial cube $X X_{1} X_{12} X_{2} X_{3}^{\prime} X_{13}^{\prime} X_{123}^{\prime} X_{23}^{\prime}$ are planar. For example, the face $X X_{2} X_{23}^{\prime} X_{3}^{\prime}$ is planar since the lines $X_{3} X_{23}$ and $X_{3}^{\prime} X_{23}^{\prime}$ meet in $A_{2}^{3}$ by construction and this is also the point of intersection of the lines $X_{3} X_{23}$ and $X X_{2}$.

Now, consider the points

$$
\begin{aligned}
A_{2}^{1} & =X X_{2} \cap X_{1} X_{12}, & & A_{3}^{\prime 1}=X X_{3}^{\prime} \cap X_{1} X_{13}^{\prime}, \\
A_{23}^{\prime 1} & =X_{3}^{\prime} X_{23}^{\prime} \cap X_{13}^{\prime} X_{123}^{\prime}, & & A_{32}^{\prime 1}=X_{2} X_{23}^{\prime} \cap X_{12} X_{123}^{\prime} .
\end{aligned}
$$

They belong to the intersection of the planes $X X_{2} X_{23}^{\prime} X_{3}^{\prime}$ and $X_{1} X_{12} X_{123}^{\prime} X_{13}^{\prime}$ and therefore are collinear. Hence, the points $A_{2}^{1}, A_{3}^{1}, A_{23}^{1}, A_{32}^{1}$ which are the projections of these points from $O$ onto $\alpha$ are also collinear. The collinearity of the points $A_{1}^{2}, A_{3}^{2}, A_{13}^{2}, A_{31}^{2}$ is proved analogously.

Notice that 8 vertices and 12 sides of the cube, 12 intersection points and 3 lines of intersections form a configuration with the symbol $\left(20_{3} 15_{4}\right)$. This configuration is regular, that is, all points and lines are on an equal footing. For example, the lines $A_{2}^{1} A_{23}^{1}$ and $A_{1}^{2} A_{13}^{2}$ correspond to the edges of the combinatorial cube with the opposite faces $X_{2} A_{2}^{1} X_{1} A_{1}^{2}$ and $X_{23} A_{23}^{1} X_{13} A_{13}^{2}$, while the lines $X X_{3}$ and $X_{12} X_{123}$ play the role of the lines of intersections for this cube. This configuration was mentioned on pp. 143 and 202 of [10], in connection with the following statement (which is equivalent to Theorem 4):

Theorem 5 (Levi). Let three triangles be perspective with the common center. Then three axes of perspective of three pairs of the triangles meet in one point.

The proof of Theorem 4 makes obvious the link between the mapping (8) and the notion of quadrilateral lattices introduced in [3]. Recall that the $M$-dimensional quadrilateral lattice is a mapping $X: \mathbb{Z}^{M} \rightarrow \mathbb{R}^{d}, d>2$, such that the image of any unit square in $\mathbb{Z}^{M}$ is a planar quadrilateral $X X_{i} X_{i j} X_{j}$. The image of any unit cube is a combinatorial cube with planar faces. It is clear that the vertex $X_{123}$ in such a figure is uniquely defined as the intersection of the planes

$$
X_{123}=X_{1} X_{12} X_{13} \cap X_{2} X_{12} X_{23} \cap X_{3} X_{13} X_{23}
$$

and therefore the three-dimensional quadrilateral lattice is reconstructed from three twodimensional ones corresponding to the coordinate planes which play the role of the initial data. The main property of this mapping proved in [3] is its four-dimensional consistency which guarantees that the $M$-dimensional lattice is also reconstructed from two-dimensional ones without contradiction. 
Accordingly to Theorem 4, the projection of the quadrilateral lattice from $\mathbb{R}^{d}$ onto a plane is reconstructed from the images of the initial data by applying the mapping (8) instead of (9). Since the quadrilateral lattice in $\mathbb{R}^{d}$ is four-dimensionally consistent, the mapping (8) is four-dimensionally consistent as well. In particular, this is also true for the reduction of the mapping (8) to a conic section, that is, for the double cross-ratio equation (6).

From all the above, the following definition of the quadrilateral lattices on the plane can be issued.

Definition. An $M$-dimensional quadrilateral lattice on the plane is a mapping $X: \mathbb{Z}^{M} \rightarrow$ $\mathbb{R}^{2}, M \geq 3$, such that the images of the corresponding edges of any pair of the opposite faces of any unit cube in $\mathbb{Z}^{M}$ meet on a straight line.

\section{The Hietarinta Equation}

Consider the partial difference equation in $\mathbb{Z}^{2}$ introduced by Hietarinta in his recent paper [4] (up to the change $x \rightarrow-x$ ):

$$
\left(x-e^{2}\right)\left(x_{1}-o^{1}\right)\left(x_{2}-e^{1}\right)\left(x_{12}-o^{2}\right)=\left(x-e^{1}\right)\left(x_{1}-e^{2}\right)\left(x_{2}-o^{2}\right)\left(x_{12}-o^{1}\right)
$$

or, as the multi-ratio,

$$
\left(x, e^{2}, x_{1}, o^{1}, x_{12}, o^{2}, x_{2}, e^{1}\right)=1 .
$$

Here $e^{i}, o^{i}$ are parameters of the equation.

Lemma 3 provides the geometric interpretation of this equation as the particular case of Theorem 2 at $N=4$. Like for the double cross-ratio equation, it is convenient to reformulate this case as a mapping from seven points into one point on a conic section $\mathcal{C}$. This mapping is illustrated by Fig. 5 and is described as follows.

Let $X, X_{1}, X_{2}$ and $O^{1}, O^{2}, E^{1}, E^{2}$ be points on $\mathcal{C}$. Then the point $X_{12} \in \mathcal{C}$ is defined by formulae

$$
\begin{gathered}
A=X O^{1} \cap X_{1} E^{1}, \quad B=X O^{2} \cap X_{2} E^{2}, \\
A^{\prime}=X_{2} O^{1} \cap A B, \quad B^{\prime}=X_{1} O^{2} \cap A B, \\
X_{12}=E^{1} A^{\prime} \cap E^{2} B^{\prime} .
\end{gathered}
$$

The corresponding values of the rational parameter on $\mathcal{C}$ are related by (10).

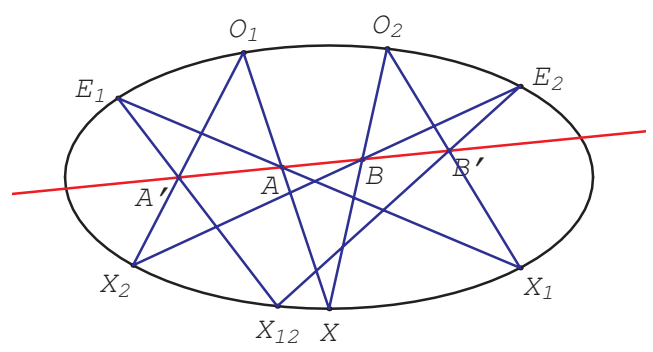

Fig. 5. The mapping (11). 
Equation (10) is three-dimensionally consistent, that is, the mapping $x: \mathbb{Z}^{M} \rightarrow \mathbb{C P}^{1}$ governed by the equation

$$
\left(x, e^{j}, x_{i}, o^{i}, x_{i j}, o^{j}, x_{j}, e^{i}\right)=1
$$

for any two-dimensional sublattice is computed from the initial data on the coordinate axes without contradiction. This can be easily checked directly. At the moment it is not clear if this property is inherited from some more general construction, as in the case of the double cross-ratio equation, and any geometric proof is not known.

\section{Acknowledgment}

I thank A. I. Bobenko and Yu. B. Suris for fruitful discussions and many useful suggestions.

\section{References}

1. V.E. Adler, A.I. Bobenko, and Yu.B. Suris. Classification of integrable equations on quad-graphs. The consistency approach. Comm. Math. Phys. 233:513-543, 2003.

2. A.I. Bobenko, D. Matthes, and Yu.B. Suris, Nonlinear hyperbolic equations in surface theory: integrable discretizations and approximation results. Algebra i Analiz 17:53-83, 2005.

3. A. Doliwa and P.M. Santini. Multidimensional quadrilateral lattices are integrable. Phys. Lett. A 233:265372, 1997.

4. J. Hietarinta. A new two-dimensional lattice model that is "consistent around a cube". J.Phys. A 37(6):L6773, 2004.

5. B.G. Konopelchenko and W.K. Schief. Three-dimensional integrable lattices in Euclidean spaces: conjugacy and orthogonality. Proc. Roy. Soc. London Ser. A 454(1980):3075-3104, 1998.

6. B.G. Konopelchenko and W.K. Schief. Reciprocal figures, graphical statics and inversive geometry of the Schwarzian BKP hierarchy. Stud. Appl. Math. 109(2):89-124, 2002.

7. F.A. Möbius. Verallgemeinerung des Pascal'schen Theorems das in einen Kegelschnit beschriebene Sechseck betreffend. J. Reine Angew. Math. 36:216-220, 1848

8. F.W. Nijhoff and A.J. Walker. The discrete and continuous Painlevé hierarchy and the Garnier system. Glasgow Math. J., 43A:109-123, 2001.

9. J.J.C. Nimmo and W.K. Schief. An integrable discretization of a (2+1)-dimensional sine-Gordon equation. Stud. Appl. Math. 100(3):295-309, 1998.

10. F. Levi. Geometrische Konfigurationen. Leipzig, 1929.

Received September 30, 2004. Online publication August 4, 2006. 